\title{
Analysis of Site Selection of Medical Centers from a Passive Defense Perspective in Omidiyeh City, Iran
}

\author{
Ali Zangiabadi ${ }^{1} \mathbb{D}$, Hojiat Bahmaei ${ }^{2} \mathbb{D}$, Seyed Ali Marashi-Shoushtari ${ }^{3}$
}

Date of submission: 27 Feb. 2019, Date of acceptance: 13 July 2019

\begin{abstract}
INTRODUCTION: Medical centers are directly involved in health of the individual and society and quick and cheap access to these centers in urban communities is of great importance. In providing urban services, considering some principles, including the principles of passive defense in structures, increases the efficiency of the urban system in the area of provision of better services to citizens and also the development of a sustainable city. This study aims to address the spatial analysis of medical centers in Omidiyeh City, Iran, based on the passive defense principles.

METHODS: The present study was applied in terms of purpose and descriptive-analytical in terms of nature and method. The data collection method included the library and survey studies. The technique used in the analysis was also the analytic hierarchy process (AHP) based on the experts' opinion and a pairwise comparison between the indicators in the geographic information system (GIS).

FINDINGS: Combining the layers of population density, land use, communication network, medical centers, distance from industrial centers, and distance from fire stations as well as creating the final map, a suitable area for medical centers was identified.

CONCLUSION: The study findings indicated that the distribution pattern of the health centers in Omidiyeh is not in accordance with the principles and criteria of passive defense and the establishment of new urban medical centers using the GIS capabilities in the city can be useful in providing services to citizens.
\end{abstract}

Original Article

Keywords: Passive Defense; Medical Centers; Site Selection; Omidiyeh; Analytic Hierarchy Process (AHP)

How to cite this article: Zangiabadi A, Bahmaei H, Marashi-Shoushtari SA. Analysis of Site Selection of Medical Centers from a Passive Defense Perspective in Omidiyeh City, Iran. Sci J Rescue Relief 2019; 11(3): 154-62.

\section{Introduction}

A review of the history of wars and conflicts, especially in recent centuries, shows that threats have developed from the military and the front lines to other areas (1). Investigating the history of the recent wars in the world, the history of 8 years of holy defense of Iran and the Israel's attacks in the 33-day war against Lebanon indicate the widespread invasion against health centers by the enemies to disable them and create a widespread crisis (2).
In the present era, the passive defense knowledge as one of the most novel defense sciences has always been of interest to scientific and military circles, to the extent that powerful countries have placed more importance on this issue. Passive defense refers to a set of measures that do not require the application of warfare and its implementation can prevent financial losses to vital and sensitive military and non-military equipment and facilities, in addition to preventing human casualties or reducing the extent of these losses and casualties to minimum (3). One of the

\footnotetext{
1- PhD, Department of Geography and Urban Planning, School of Geography, University of Isfahan, Isfahan, Iran

2- MSc, Geography and Urban Planning, School of Geography, University of Isfahan, Isfahan, Iran

3- MSc, Passive Defense, Malek Ashtar University of Technology, Tehran, Iran

Correspondence to: Hojjat Bahmaei, Email: hojjat88@ymail.com
} 
essential principles of passive defense is site selection, as without a proper site selecting, other defense considerations are practically ineffective or less effective. Site selection can be defined as finding a suitable place for a defense center, special facilities, an industrial zone, etc. in such a way that different parameters such as the shape of the area, distance from communication routes, distance from population centers, etc. with different weights are involved in finding it (4).

The city of Omidiyeh, Iran, which was examined in this study, has some shortcomings in terms of health care service centers. The number of health centers in this city (one hospital, three clinics, and an emergency center) is not in a good position according to some common standards, namely the necessity of establishment of a hospital for every 45,000 to 50,000 people (5) considering the 91,000 population of the city based on the 2011 National Population and Housing Census. However, the city is in almost good condition in terms of distribution and access radius, which affect the vulnerability of the urban areas. The main purpose of this study is to find the indicators related to the site selection of medical centers, their prioritization, and their application in the study case of the Omidiyeh City. The secondary objective of the study is to assess the level of compliance of the medical centers existing in Omidiyeh City in terms of distribution and area of function with global standards.

Although the provision of health care services in cities has a long history, there is no long-term history in locating health care centers, and such studies date back to the 1970s. Overall, no study has been accomplished on human crisis management with a passive defense approach in Omidiyeh and the few studies performed in this city have addressed crisis management in natural areas such as floods and earthquakes. In this section, some of the internal and international studies conducted in connection with this issue are as follows:

In a study entitled "site selecting with passive defense considerations in the GIS environment" Tavakoli Zadeh et al. evaluated the important principles of passive defense in crisis management and site selection of optimal areas for post-crisis reorganization and reconstruction. The results of this study suggested that the protection and preservation of important resources, facilities, and equipment in a region or city before the crisis and the proper site selecting for their transfer after the crisis can be very effective in reducing damages (6).

Mohammadpour and Zarghami examined and introduced the requirements for locating urban facilities from a passive defense perspective. They briefly reviewed the models and techniques used in locating urban facilities, and introduced four models of Boolean logic, index overlay (IO), fuzzy logic, and weights-of-evidence. Additionally, they expressed the passive defense considerations in the design and planning of the urban infrastructure facilities, including water and sewage facilities, water treatment plants, gas facilities, electronic facilities, and site selection of fire stations, telecommunications, and medical centers for urban development. This study was of a descriptive type and provided a general overview of site selecting of urban facilities (7).

Ferdowsi et al. in their study as "Hospital site selection using passive defense approach" investigating the criteria for hospital site selection concluded that the hospital sites should be safe and in the neighborhood of compatible uses and away from incompatible uses. This study showed that in locating a hospital with a passive defense approach, factors such as the hospital's location (away from dangerous areas), access to highways and subways, proximity to densely populated areas, urban distributions, proximity to green spaces and open spaces and fire centers, and distance from faults and rivers must be considered (8).

Hoseini et al. carried out a study on "The site selection of hospital centers on passive defense approach using colonial competitive algorithm (case study: District 3 of Tehran, Iran)" taking into account three principles of safety, adaptability, and efficiency, and selecting the subcriteria of pollution, population density, roadblocks, access to roads, proportionality, proximity, and service area. They concluded that the site selecting of hospitals in District 3 of Tehran was not in line with the principles of passive defense, and proposed six new sites for hospital construction (9).

In a study entitled "Modeling hospital site section by using Fuzzy logic by combining AHP and TOPSIS in ARCGIS environment", with four criteria of adaptability, desirability, capacity, and layer of constraints, Pourahmad et al. assessed the 
compatibility of land in Tabriz, Iran, for hospital construction (10).

Grundy et al. in a study entitled "The role of public health as a lever of passive defense in war" concluded that paying attention to the health actions of public colleagues before the crisis and war will help plan and structure in war and leads to successful planning (11).

Shishebori et al. in a study as "Robust site selection of hospitals with passive defense by integrated AHP-TOPSIS method (Case study; Amol County)" after determining all important criteria of sustainability and passive defense and important criteria for determining the location of a new hospital, through a questionnaire and using the opinion of experts, concluded that in addition to passive defense criteria, sustainability criteria are also important (12).

In a study entitled "Spatial analysis and site selection of health medical and hospital centers using (GIS) (Case study: Jahrom city)", Sahraeian et al. concluded that the use of GIS system with AHP, given its special characteristics can be applied in the study of urban planning issues. Moreover, by examining the case study, they found that the distribution of medical centers in Jahrom City was associated with shortcomings, so they suggested new places to create these centers (13).

\section{Methods}

The present study was a descriptive-analytical study in terms of method, in which the data collection tools included library and internet resources, field observation, and questionnaire. This project included four steps.
1. Determining the main indicators effective in locating the hospital with the help of experts, obtaining six main indicators: layer of communication network, layer of existing medical centers, layer of land use, layer of population density, distance from industrial centers, and distance from existing fire stations.

2. Weighing the indicators by the AHP method

3. Creating layers related to each index by network analysis method and using ArcGIS software

4. Combining the layers and determining the appropriate site for the construction of the hospital

The study process is illustrated in Figure 1. In order to locate the new hospital in Omidiyeh City, it was necessary to study the various capabilities of urban spaces in terms of the presence of suitable and sufficient land and its connection with the network of passages and other urban uses and facilities, especially detection of areas requiring such services. Therefore, due to the large amount of information required to locate hospitals and due to the spatial nature of the data, the spatial analysis tools such as geographic information system (GIS) and multi-criteria decision-making (MCDM) models such as AHP were used.

The need to use the AHP method: AHP is one of the most effective decision-making techniques that was first proposed by Thomas L. Saati in 1980. This model is based on the pairwise comparisons and allows managers to examine different scenarios. In evaluating any subject, we need measuring criteria with indices, and choosing the right index allows us to make a correct comparison between the alternatives.

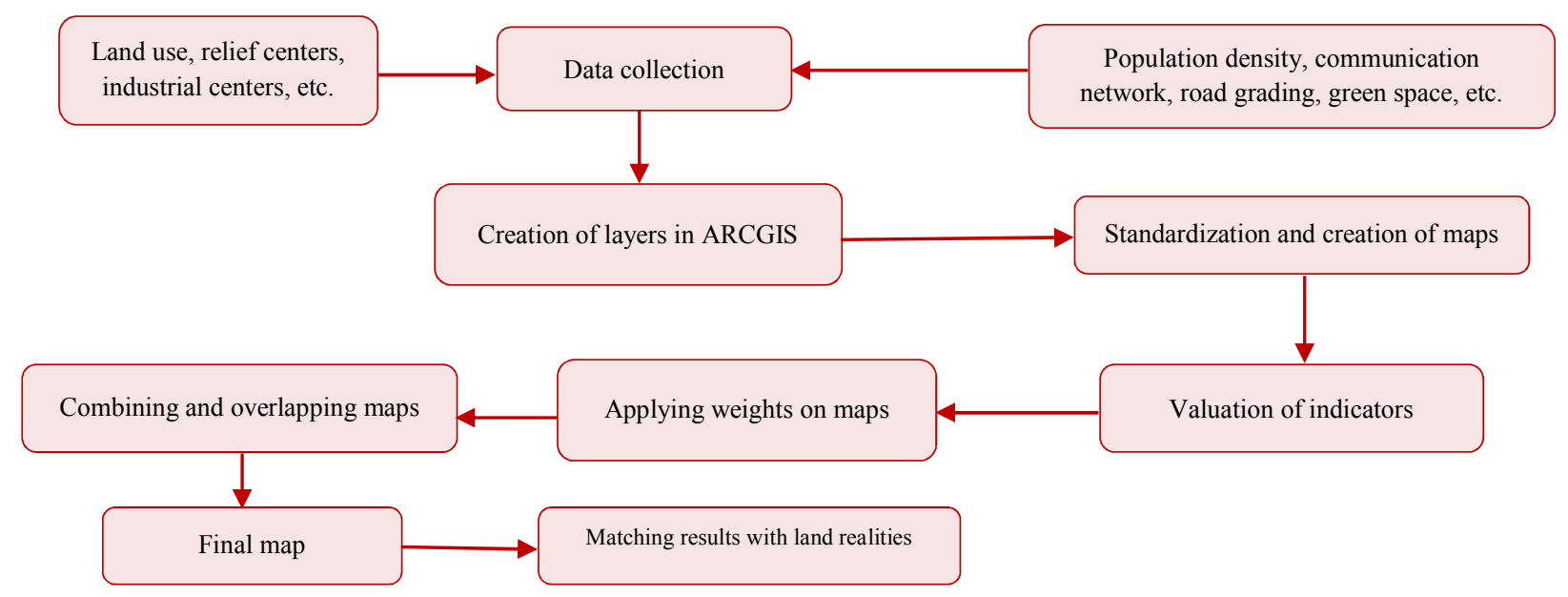

Figure 1. Study process 
However, when several or more options are together in space and of different types, then the evaluation and comparison tasks change from the simple analytical state that the mind is able to perform into a powerful practical analysis tool will be needed. One of the powerful tools for such situations is AHP. This method is used for grading, and may sometimes be used for social and economic analysis. In this method, the data of each site selection must be standardized primarily.

The AHP modeling process begins with identifying elements and making decisions and prioritizing them. These elements include different methods of performing tasks and prioritizing criteria or characteristics. In general, this method consists of three general steps described as follows:

Step 1: This includes preparation of data and pairwise matrix, which is performed by considering a scale of nine quantities proposed by L. Saati to give weights to the criteria from 1 to 9 based on their importance.

Step 2: Calculation of the final weight of the criteria, for which the numbers of each column and row are multiplied by each other, then the products of the weights are given a power of $1 / \mathrm{N}$, and finally, to calculate the final weight of the criteria, the non-normalized weights of each row are divided by the total non-normalized weights. The sum of the total final weights must be equal to 1 .

Step 3: Obtaining the consistency ratio (CR) that includes four stages.

A: Calculating AW: to determine the value of the vector, each of the weights must be multiplied by the value of the standard weight.

B: Calculating consistency vector

A: Calculating the consistency index $(\mathrm{CI})$

D: Calculating CR $(14,15)$.

After calculating the weight of each criterion, ArcGIS software was used to apply them to the maps. For this purpose, first, a network of all communication routes in the city under study, along with other features of the routes [street type (arterial, collector, distributor, and local access)], length and width of the routes, one-way, two-way, and closed routes, traffic volume, average speed, and the intersections and the existing constraints are prepared and a spatial relationship is created among the network lines through which the functional radius of each hospital can be obtained. Buffer command is used in Arc GIS software to check the status of access to critical centers, including medical centers.

The Omidiyeh map was prepared based on the actual traffic directions and after creating a topology in the Auto Map environment, other complementary network information was added and the network was intelligent, according to which the time factor, ie the duration of each route, was obtained. The Buffer command was used in Arc GIS software to examine the level of access to critical centers, including medical centers.

The map of the Omidiyeh City was prepared based on the actual traffic directions, and after creating a topology in the Auto Map environment, other complementary network information was added and the network became intelligent and accordingly, the time factor, i.e. the time for each route was obtained and based on which the functional area of each hospital at the city level was obtained.

\section{Findings}

Identification of effective parameters in site selection: Identifying the factors affecting the site selection is an important step in the study (16), so in order to optimally locate hospitals, it was tried to identify and investigate different parameters and indicators that could somehow be effective in the site selecting process in the form of following layers as much as possible. It should be noted that all the following indicators have been selected according to their impact on passive defense.

1. Layer of communication network, 2. Layer of existing medical centers, 3. Layer of land use, 4. Layer of population density, 5. Distance from industrial centers, and 6. Distance from existing fire stations.

Valuation of information layers: Evaluation refers to the weighting of the information layers used in proportion to their importance and impact on site selection. In this study, AHP model was employed to weigh the information layers. In this method, weighting is performed according to the criteria in Expert Choice software, which was implemented as follows:

A: Creating the pairwise comparison matrix: This method uses a basic scale with values from 1 to 9 to determine the relative preference of two criteria. In this matrix, if the preference of criterion $\mathrm{A}$ is twice as high as criterion $\mathrm{B}$, criterion $\mathrm{B}$ is preferable as half as criterion A. It should be noted that comparing each criterion with itself leads to a score of 1 (equivalent preference). Therefore, the number one is considered in the main diagonal of the matrix (15). 
Table 1. Pairwise comparison matrix of hospital site selection criteria

\begin{tabular}{lcc|cc|cc|ccc} 
Information & $\begin{array}{c}\text { Final } \\
\text { weight }\end{array}$ & $\begin{array}{c}\text { Fire } \\
\text { stations }\end{array}$ & $\begin{array}{c}\text { Green } \\
\text { space }\end{array}$ & $\begin{array}{c}\text { Industrial } \\
\text { centers }\end{array}$ & $\begin{array}{c}\text { Medical } \\
\text { centers }\end{array}$ & $\begin{array}{c}\text { Land } \\
\text { use }\end{array}$ & $\begin{array}{c}\text { Communication } \\
\text { network }\end{array}$ & $\begin{array}{c}\text { Population } \\
\text { density }\end{array}$ \\
\hline $\begin{array}{l}\text { Population density } \\
\text { Communication }\end{array}$ & 0.316 & 4.2 & 4 & 3.5 & 3 & 2.5 & 2 & 1 \\
network & 0.219 & 3.4 & 3.20 & 2.8 & 2.40 & 1.9 & 1 & 0.50 \\
Land use & 0.150 & 2.6 & 2.40 & 1.2 & 1.80 & 1 & 0.52 & 0.40 \\
Medical centers & 0.109 & 2.1 & 1.90 & 1.7 & 1 & 0.55 & 0.41 & 0.33 \\
Industrial centers & 0.083 & 1.7 & 1.60 & 1 & 0.58 & 0.47 & 0.35 & 0.28 \\
Fire stations & 0.056 & 1 & 0.66 & 0.58 & 0.47 & 0.38 & 0.29 & 0.23 \\
Total & 1 & 16.5 & 14.76 & 12.30 & 9.77 & 7.21 & 4.88 & 2.99 \\
\hline
\end{tabular}

B: Calculating the weight of the criteria: This step consists of the following operations:

A. Adding up the values of each column of the pairwise comparison matrix.

B. Dividing each component of the matrix into its corresponding column (the resulting matrix is called the normalized pairwise comparison matrix).

C. Calculating the average of the components in each row of the normalized matrix, i.e. dividing the sum of the normalized scores on each row by the number of criteria. This average is an estimate of the relative weight of the criteria compared. Table 1 shows an example of the matrices created for the location criteria of emergency bases.

A: Estimating CR: At this stage, if the comparisons made are consistent, it will be determined. This stage involves the following operations: Determining the weighted sum vector (WSV) by multiplying the weight of the first criterion by the first column of the main pairwise comparison matrix, then multiplying the second criterion in the second column, and this is performed for all criteria, finally adding these values in the rows and determining the vector of agreement by dividing WSV by the weight of the criteria: $L=\frac{1}{N}\left[\sum_{I=1}^{N}\left(\frac{A W}{W I}\right)\right]$

After calculating the vector of agreement, it was necessary to calculate the values of the other two terms, Lambda $(\lambda)$ and $\mathcal{C J}$. The value of $\lambda$ is equal to the average of the consistency vector values. The calculation of $\mathcal{C J}$ is based on the fact that $\lambda$ is always greater than or equal to the number of criteria under study (n), and $\lambda=n$ if the pairwise comparison matrix is consistent. Therefore, $\lambda-n$ can be considered as a criterion of the degree of consistency which is determined as follows: $\mathcal{C J}=\frac{\lambda-n}{n-1}$

The term CI, referred to as the consistency index, is a criterion for deviating from the consistency.

$\mathrm{CR}$ can also be calculated as follows: $\mathcal{C R}=\frac{\mathcal{C J}}{\mathcal{R J}}$
Where $\mathcal{R} \mathcal{J}$ is a random index of a pairwise comparison matrix generated by agreement. It can be shown that $\mathcal{R} \mathcal{J}$ depends on the number of components being compared. $\mathcal{C}$ R is designed in such a way that if $\mathcal{C R}<0.1$, it shows the acceptable level of agreement (consistency) in the pairwise comparisons, but if $\mathcal{C} R \geq 0.1$, it indicates inconsistent judgments. In such cases, the main values of the pairwise comparison matrix should be reconsidered and modified $(14,15)$.

CR was calculated for each criterion as described, which was less than 0.1 in all cases, indicating the acceptability of the pairwise comparisons.

Table 2 illustrates the weighting steps of the information layers in the AHP model for locating hospitals. As can be seen from the table above, for the information layers, distance from green space, distance from fire stations, and distance from the main communication network are given lower and higher scores by increasing and decreasing the distance, respectively.

\section{Discussion and Conclusion}

The area of function of hospitals and health centers in the city is a range the emergency base of which is able to cover in a specific and standard time for relief. The standard time for an ambulance to reach the accident scene is 3 minutes according to the world standard (17). Although this distance is usually considered to be between 1.5 and $2.5 \mathrm{~km}$ travelled at an average speed of $30 \mathrm{~km} / \mathrm{h}$ (18), this distance varies depending on factors such as the texture of the city, the structure of the communication network and traffic situation, etc., and can be more or less. Therefore, in order to determine the functional area and the area under service with the global standard time ( 3 minutes), the Find Service Area operation was used in the network analysis model. 
Table 2. Steps of weighting criteria and sub-criteria in the analytic hierarchy process (AHP) model for hospital location

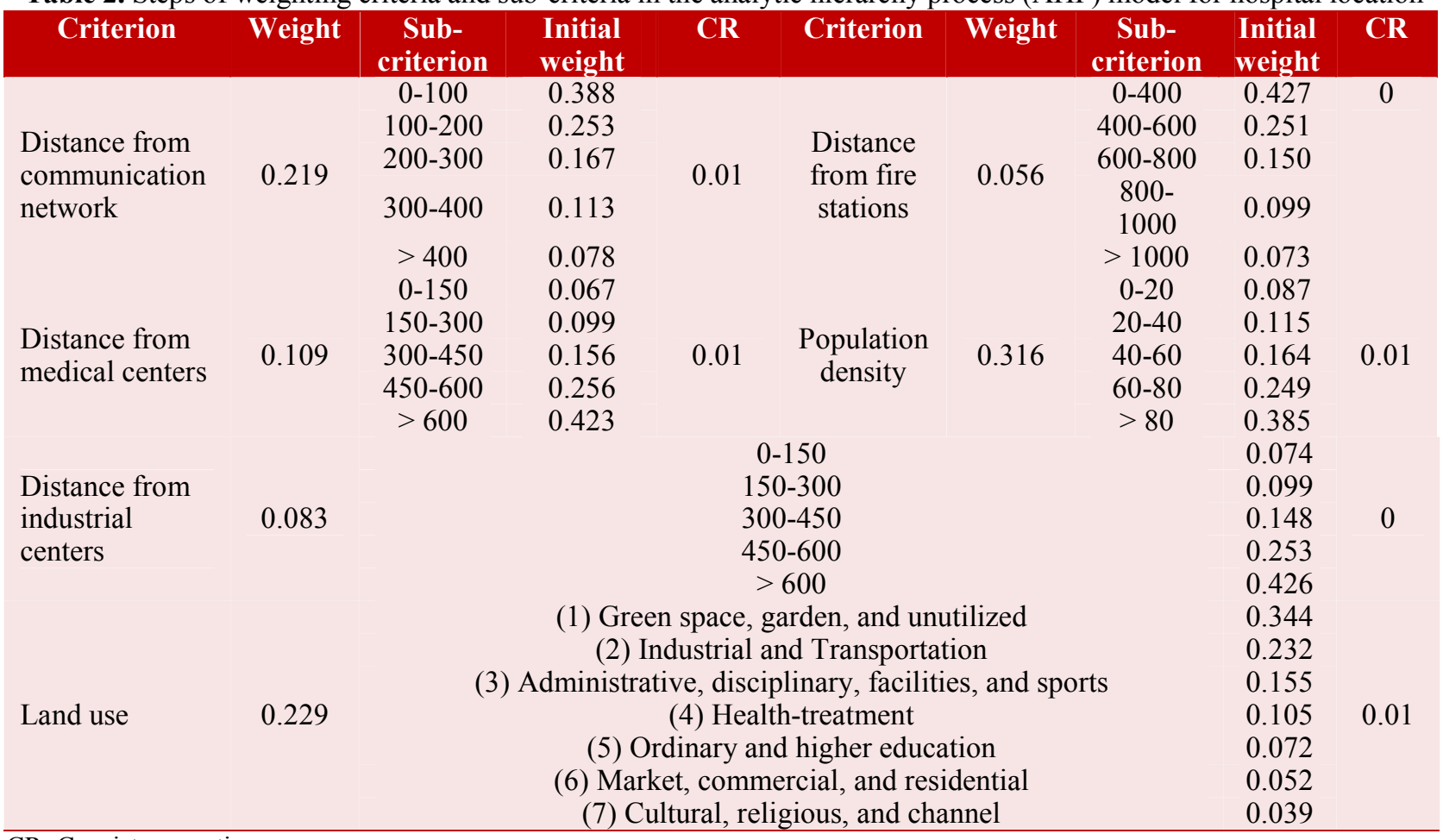

Omidiyeh City has one hospital and four health centers in which the Sherkati region has the best condition in terms of the number of medical centers with one hospital and two clinics. To assess the vulnerability level based on the principle that the more accessible the urban areas to the vital centers of the city, the less vulnerable they are, as well as the functional area of each hospital, which is $2 \mathrm{~km}$ according to current standards, the functional area of each center was determined by the hospital and the health center separately (Figure 2).

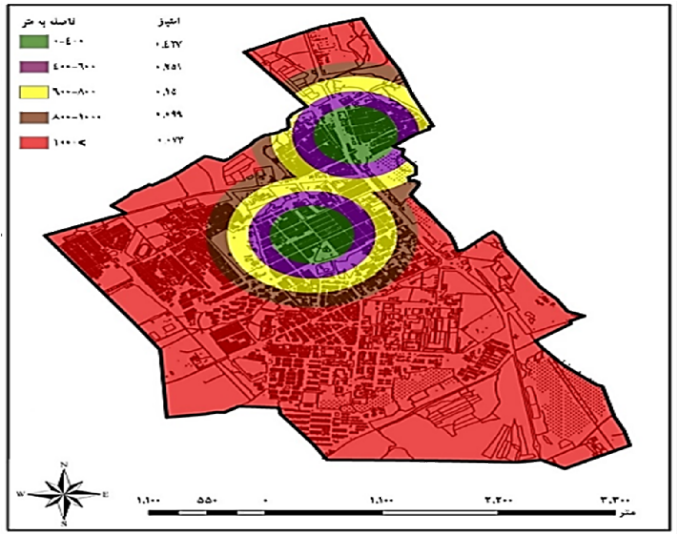

Figure 2. Distance and scoring by distance from medical centers (Reference: Municipality of Omidiyeh, Iran)
In the layer of distance from fire stations, the greater the distance between the construction site of the hospital and the fire station, the lower its score (Figure 3).

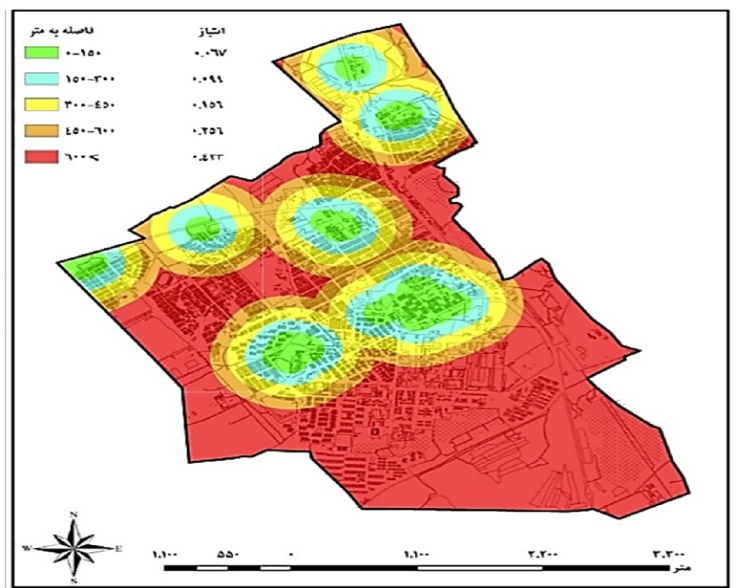

Figure 3. Distance and scoring by distance from fire stations (Reference: Municipality of Omidiyeh, Iran)

In the layer of distance from the communication networks, the more access the hospital construction site has to the main arteries, the higher the score (Figure 4). In the layer of distance from industrial centers, by increasing and decreasing the distance, respectively more and less scores were given (Figure 5). 


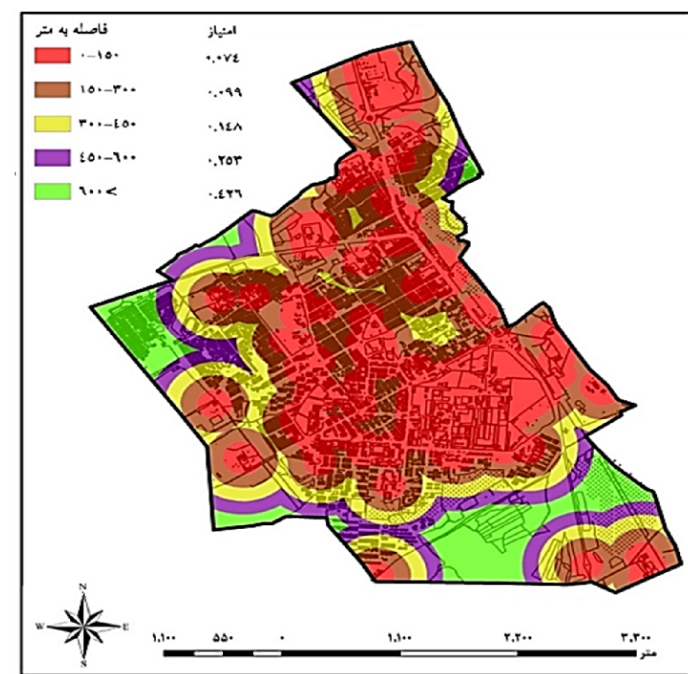

Figure 4. Distance and scoring by distance from main communication networks (Reference: Municipality of Omidiyeh, Iran)

Since high-density urban areas are more likely to be damaged than the low-density areas, establishing medical centers close to the more populated areas is of higher priority.

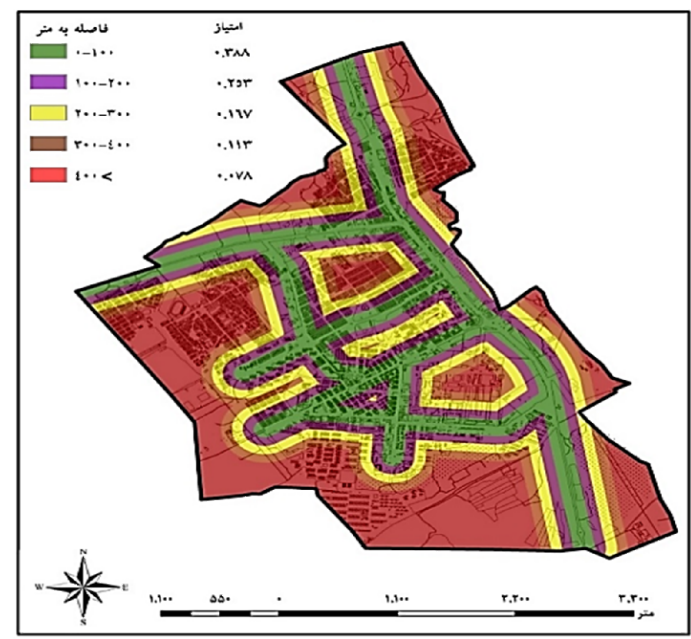

Figure 5. Distance and scoring by distance from industrial centers (Reference: Municipality of Omidiyeh, Iran)

Thus, given that one of the goals of site selection of the health care centers is to make most of the population benefit from these centers, the population density layer was scored; In this way, neighborhoods with higher and lower density received more and less scores, respectively (Figure 6). For the land use layer, scores were considered based on the economic value and the proportion of lands for the establishment of health centers.

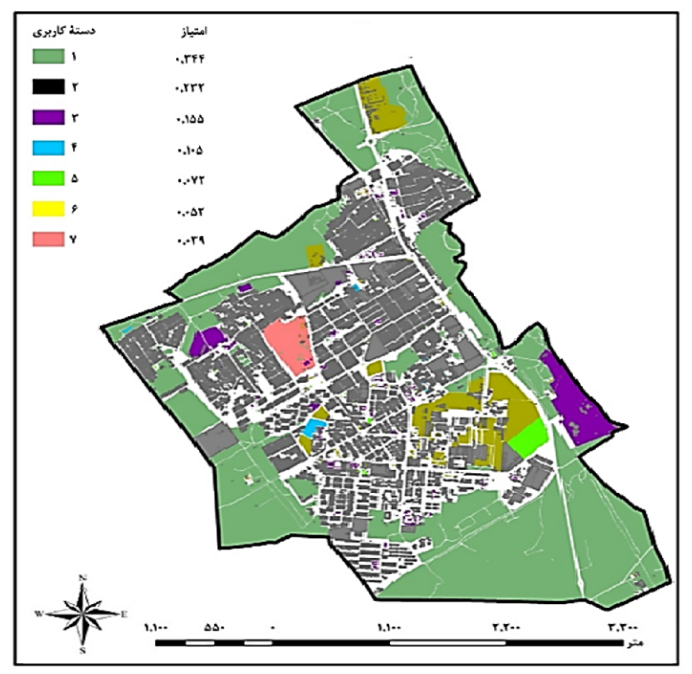

Figure 6. Scoring of population density of neighborhoods (Reference: Municipality of Omidiyeh, Iran)

For example, the unutilized land use was given a higher score on the basis of lower economic value and the commercial use was given a lower score on the basis of higher economic value (Figure 7).

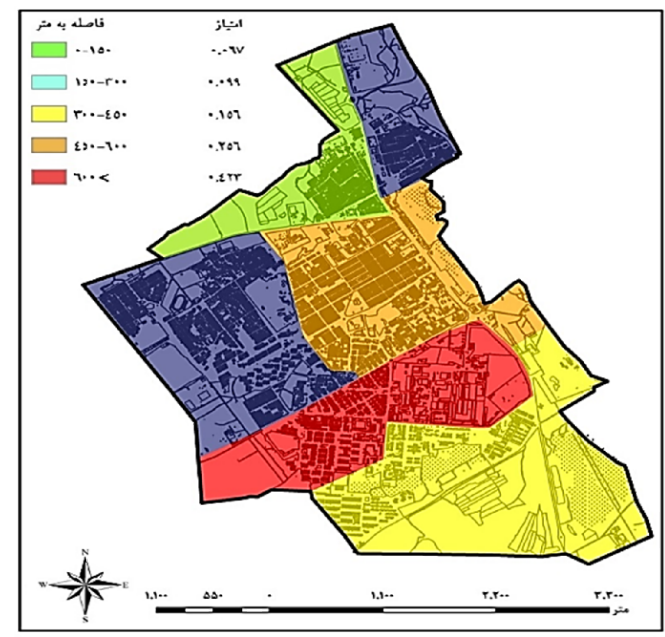

Figure 7. Land use scoring (Reference: Municipality of Omidiyeh, Iran)

Combination of information layers (preparation of the final scoring map): The combination of layers and the preparation of the final map are commonly performed according to the project requirements and usually in several different ways. If the purpose of the site selection is only to determine the appropriate locations with a high degree, these maps are prepared in the form of single-purpose maps and only with the ability to display the appropriate areas. In such maps, there is no ranking (different zones) among the parameters. 
In another form of the final maps, in addition to determining the appropriate locations, locations with low appropriateness to inappropriate locations are prepared depending on the project requirements. These types of maps usually show the zones separately (color, symbol, etc.) (19). In this study, the IO model was employed to combine the layers with each other, with the results demonstrated in Figure 8. At this stage, the categories of each layer were weighted in accordance with the obtained rank and using the Raster Calculator tool, the score columns related to each of the information layers created were added together.

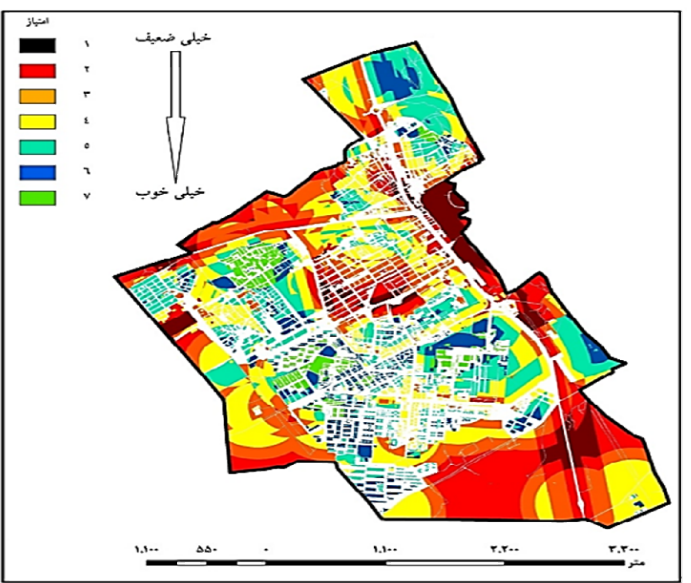

Figure 8. Final scoring of urban lands for the establishment of an emergency base (Reference: Municipality of Omidiyeh, Iran)

At this stage, the final map was scored by classifying the data into 7 distinct classes, from highly inappropriate to highly appropriate, to establish new hospitals. In this way, it is possible to change the uses, determine the appropriate areas, and locate new sites to construct new hospitals in the city of Omidiyeh, and so on.

In the present study, by examining the effectiveness of the indicators affecting the location, the following prioritization can be concluded:

1. The population density layer, 2. The land use layer, 3 . The communication network layer, 4 . The existing medical centers layer, 5. The distance from the industrial centers, 6. The distance from the existing fire stations.

Matching the location model results with the land realities: One of the most important issues to consider after selection and location by GIS is how well the specified areas match the realities and conditions of the area. To investigate this issue, field observations and studies can show the accuracy of the located areas (20). The more the identified factors for the location match the terrestrial reality, the more satisfactory the location results will be (16). After matching the results of the location model with the reality in the study area and taking into account all the effective parameters in the process of locating and calculating the service range of 3 minutes and 5 minutes and according to the final scoring map (Figure 9), three new emergency stations were proposed for the city of Omidiyeh.

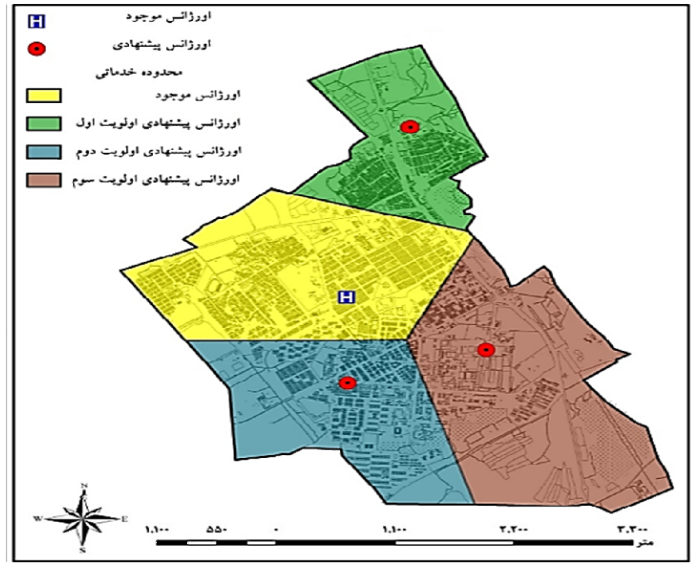

Figure 9. Locations proposed for establishing new emergency bases (Reference: Municipality of Omidiyeh, Iran)

\section{Solutions}

- Locating fire stations in the right range of vital, sensitive, and important urban uses due to the effective role of these centers in reducing vulnerability during crises and especially wars.

- Locating new hospitals in different parts of the city taking into account the principles and criteria of passive defense and avoiding their concentration in urban centers, especially in busy areas.

- Proximity of public open spaces and medical centers in the city due to the complementary role of these two uses in times of crisis, as well as the adoption of programs to expand appropriate open spaces within the hospital grounds.

- Locating new medical centers according to the main arteries of the city in such a way that medical centers can be accessed in the shortest possible time and with the least traffic.

- Using the results of the proposed maps in prospective planning as well as in management decisions. 
- More attention of managers to the principles of passive urban defense and its inclusion in comprehensive urban plans.

- Using GIS and its capabilities in planning services and management issues.

- The necessity to pay attention to the establishment of new medical centers in the future in accordance with the principles of passive urban defense based on the factors examined in this study.

- Modification of some centers as well as the creation of several other small centers in some cases.

- Elimination of existing obstacles, especially in terms of access and urban context for faster response of medical centers in cases where these factors are inappropriate and restrictive.

\section{Acknowledgments}

The authors of the study would like to appreciate all those who have contributed to this study.

\section{Conflict of Interests}

Authors have no conflict of interests.

\section{References}

1. Movahedinia J. Principles of passive defense. Tehran, Iran: Malek Ashtar University of Technology Publications; 2009. [In Persian].

2. Piri H, Hassan Nejad H, Akbar Fakhrabadi M. Prioritizing hospital for managing construction protecting against man made threat. Journal of Engineering \& Construction Management 2016; 1(1): 33-6. [In Persian].

3. Bahmaei H. Passive defense in oil cities with emphasis on physical-spatial dimensions (case study: Omidiyeh city) [MSc Thesis]; Isfahan, Iran: Department of Geography and Urban Planning, University of Isfahan; 2013. [In Persian].

4. Dargahi H, Sadr Momtaz M, Faraji F. Hospital standards. Tehran, Iran: University of Tehran Press; 2005. [In Persian].

5. Shieh E. Introduction to urban planning. Tehran, Iran: Iran University of Science and Technology Publications; 2007. [In Persian].

6. Tavakoli Zadeh M, Setareh AA, Kheirabadi A. Site selecting with passive defense consideration in GIS field. Proceedings of the National Conference of Geographic Information System; 2009 Nov 22-23; Tehran, Iran. 2020.

7. Mohammadpoor A, Zarghami S. Buyer's locating urban facilities from the standpoint of passive defense. Geographical Data 2014; 23(90): 89-93. [In Persian].
8. Ferdowsi M, Masoud M, Nadri Fathabad S. Hospital site selection using passive defense approach. Sci J Rescue Relief 2016; 8(3): 56-69. [In Persian].

9. Hoseini SA, Ahad Nejad Ravashti M, Modiri M, Arish S. The allocation of hospital centers on passive defense approach using colonial competitive algorithm (Case: Tehran 3 District). Journal of Geography and Regional Development 2013; 11(21): 223-45. [In Persian].

10. Pourahmad A, Ashlaghi M, Ahar H, Manuchehr A, Ramazani Mehraban M. The location modeling of hospitals using Fuzzy Logic combining AHP and TOPSIS in ArcGIS environment. Geography and Environmental Planning (University of Isfahan) 2020; 25(2): 1-24.

11. Grundy J, Leslie Annear P, Mihrshahi S. Balancing national security with human security: A call for comprehensive pre-event public health analysis of war and defense policy. Journal of Peace, Conflict and Development 2008; (12).

12. Shishebori D, Asghari Akha N, Yousefi Babadi A, Salimi N. Robust location of hospitals in with passive defense by integrated AHP-TOPSIS method (Case study; Amol County). Passive Defense Quarterly 2017; 7(4): 33-46.

13. Sahraeian Z, Zangiabadi A, Khosravi F. Spatial analysis and site selection of health medical and hospital centers using (GIS) (Case study: Jahrom city). Geographic Space 2013; 13(43): 153-70. [In Persian].

14. Modiri M, Hosseini SA, Ahadnejad Rovashti M, Kameli Mofrad MJ. The assessment of urban region quality for urban utilities distribution in man-made crises with passive defense approach. Journal of Spatial Planning 2013; 3(2): 79-100. [In Persian].

15. Mahmoudzadeh H. Use of ARCGIS in urban planning. Tabriz, Iran: Elmiran Publications; 2010. [In Persian].

16. Farajzadeh M. GIS and its application in tourism planning. Tehran, Iran: Samt Publications; 2015. [In Persian].

17. Bahrami S. Network analysis of medical emergencies and services using GIS (case study: Isfahan city) [MSc Thesis]; Isfahan, Iran: School of Literature and Human Sciences, University of Isfahan; 2008. [In Persian].

18. Arseh Consulting Engineers. Research on the place and dimensions of urban fire protection. Tehran, Iran: Ministry of the Interior, Center for Urban Planning Studies; 1999. [In Persian].

19. Azimi Hosseini M, Nazari Fard MH, Momeni R. GIS application in site selection. Tehran, Iran: Mehregan Galam Publications; 2010. [In Persian].

20. Varesi HR, Mohammadi J, Shahivandi A. Locating urban green space using GIS model (case study: Khorramabad). Journal of Geography and Regional Development2008;(10):83-103.

[In Persian]. 